Bio - grafia. Escritos sobre la Biología y su Enseñanza. ISSN 2027

Edición Extraordinaria. p.p. 744 - 770

Memorias del VIII Encuentro Nacional de Experiencias en Enseñanza de la Biología y la Educación Ambiental. III Congreso Nacional de Investigación en Enseñanza de la Biología.

\title{
ELABORACIÓN DE PROTOTIPOS ECOLÓGICOS: UNA PROPUESTA EN LA CONSTRUCCIÓN DE CULTURA AMBIENTAL EN EL COLEGIO FERNANDO SOTO APARICIO DE BOGOTÁ D.C.
}

\author{
Sandra Teresa Piñeros Salgado y Carlos Humberto Barreto Tovar ${ }^{2}$
}

\section{RESUMEN}

El presente artículo describe la experiencia de las docentes de Ciencias Naturales y educación Ambiental Sandra Piñeros y Alicia González en el Colegio Fernando Soto Aparicio IED, ubicado en la localidad de Kennedy. Esta experiencia desarrollada en el marco del Proyecto Ambiental Escolar PRAE, se basa en la reutilización de residuos sólidos para la construcción de prototipos ecológicos de manera tal que se generen acciones que fortalezcan la cultura ambiental a partir de la participación de los estudiantes en los procesos de mejora de su entorno. La elaboración de prototipos en la escuela es un tema poco abordado desde esta óptica y por eso las docentes

E pretenden extender la invitación a participar activamente en procesos similares al interior de las instituciones educativas.

$\mathrm{N}$

C

I

A

This article talks about Sandra Piñeros and Alicia Gonzalez experiences in the School Enviromental Project (PRAE- acronym in Spanish). They both are Science and Enviromental Education teachers at the school named Fernando Soto Aparicio, located in Kennedy (BogotaColombia) where this Project has been implemented. This strategy intend to generate actions that results in the environmental culture strengthening with the students' participation in the process of reusing solid waste to build some ecological prototypes to improve their environment. Prototyping in school is a topic not fully understood yet and this strategy tries to extend the invitation to participate inside schools.

${ }^{1}$ Universidad de la Sabana. Facultad de Educación. Maestría en Pedagogía. Coinvestigadora. Docente de Ciencias Naturales y Educación Ambiental del Colegio Fernando Soto Aparicio IED. Correo: profesatepisa@gmail.com

2 Universidad de la Sabana. Facultad de Educación. Maestría en Pedagogía. Docente - Investigador. Correo: carloshumbertobarreto@hotmail.com 
Bio - grafia. Escritos sobre la Biología y su Enseñanza. ISSN 2027

Edición Extraordinaria. p.p. 744 - 770

Memorias del VIII Encuentro Nacional de Experiencias en Enseñanza de la Biología y la Educación Ambiental. III Congreso Nacional de Investigación en Enseñanza de la Biología.

Keywords: environmental culture, ecological prototype, environmental committee, environmental project.

\section{Introducción}

La construcción de la Cultura Ambiental en la escuela (Miranda, 2013) se constituye en uno de los retos de la educación en el presente. Formar estudiantes con cultura ambiental de manera tal que se genere el criterio y la autonomía en la toma de decisiones frente a los procesos de transformación y conservación del entorno a lo largo de su existencia, puede ser una meta, fruto de la interiorización de su misión como sujeto activo en el gran entramado de la vida del Planeta.

Es propio de las políticas educativas nacionales propender por la consolidación de un ideario ambiental en las instituciones educativas como se puede apreciar en lo dispuesto en el Decreto 1743 del 3 de Agosto de 1994 expedido por el Ministerio de Educación Nacional que afirma... "todos los establecimientos de educación formal del país, tanto oficiales como privados, en sus distintos niveles de preescolar, básica y media, incluirán dentro de sus proyectos educativos institucionales, proyectos ambientales escolares en el marco de diagnósticos ambientales, locales, regionales y/o nacionales, con miras a coadyuvar a la resolución de problemas ambientales específicos".

Estas y otras legislaciones vigentes evidencian los objetivos perseguidos desde el nivel central de gobierno, planteando la transversalidad del tema ambiental sin embargo, en el cotidiano de la escuela, el maestro no logra que los estudiantes se involucren en el diagnóstico de la problemática específica de su institución, menos aún en acciones metodológicas reales para la resolución de la misma.

Partiendo del proceso de unificación y resignificación del Proyecto Ambiental Escolar - PRAEy con la asesoría de la Dra. Paola Moreno, bióloga de la Fundación FES; se genera en el año 2012 el proyecto institucional titulado "Por un ambiente que promueva el aprendizaje y nos conecte con la naturaleza y la sociedad". Como docentes encargadas del desarrollo del PRAE en la Institución y atendiendo a la necesidad de realizar actividades que permitieran el desarrollo de conciencia ambiental, se escribió el PRAE, instrumento que brindó los lineamientos generales para que en cada sede y jornada (la institución cuenta con tres sedes en las cuales funcionan 5 jornadas) se ajustara un Plan de Acción de acuerdo al diagnóstico particular pero atendiendo a los fines y objetivos comunes de éste documento. (Colegio Fernando Soto Aparicio, IED, 2012). 
Edición Extraordinaria. p.p. 744 - 770

Memorias del VIII Encuentro Nacional de Experiencias en Enseñanza de la Biología y la Educación Ambiental. III Congreso Nacional de Investigación en Enseñanza de la Biología.

Posteriormente y como un esfuerzo por integrar el PRAE en las actividades programadas desde el área de Ciencias Naturales, se desplegó la propuesta de incluir como un componente académico dentro de las Mallas curriculares; las temáticas y competencias de la ecología, ya que permitirían desde la base conceptual orientar las actividades para cada ciclo en el tema ambiental. (Colegio Fernando Soto Aparicio, IED, 2012)

Con los estudiantes de los grados quinto y sexto, pertenecientes al ciclo 3 de Educación Básica y a partir del programa Bogotá, Basura Cero, propuesto por la Alcaldía Mayor de Bogotá y la Secretaría de Educación y teniendo en cuenta el eje Disposición de los Residuos sólidos, se implementó la estrategia de la recolección y acopio de las bolsas plásticas en las que se entrega empacado el refrigerio a los estudiantes. (Ver fig. 1).

\section{Metodología}

La actividad comenzó haciendo un análisis sobre el tipo y cantidad de residuos sólidos cotidianos y poniendo énfasis en la necesidad de que la institución se hiciera cargo de éstos desechos que a diario se producían. Se hizo un cálculo inicial teniendo en cuenta el promedio de 35 estudiantes por curso que a diario reciben refrigerio personal, este se encuentra empacado en al menos tres bolsas de plástico, lo que cual daría un total de 105 bolsas plásticas por curso, al ser consumido el refrigerio en 10 cursos, de los aproximadamente 50 cursos, se obtiene un total de 1050 bolsas diarias..

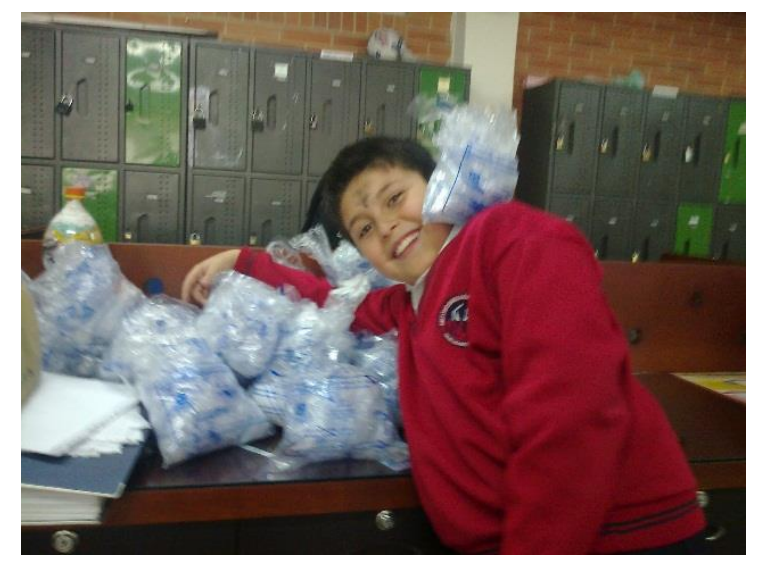

Fig.1. Recolección diaria de los empaques plásticos del refrigerio 
Edición Extraordinaria. p.p. 744 - 770

Memorias del VIII Encuentro Nacional de Experiencias en Enseñanza de la Biología y la Educación Ambiental. III Congreso Nacional de Investigación en Enseñanza de la Biología.

El plástico recolectado se convirtió en un insumo para diversas iniciativas que tanto estudiantes como profesores proponían para su aprovechamiento. La primera de estas actividades fue elaborar carteleras con mensajes alusivos a la conservación del planeta, la idea fue acogida por los estudiantes del grado 401. La recolección, acopio, clasificación y diseño de la cartelera resultó ser una actividad que además de mostrar un resultado desde lo ambiental, también permitió a través del trabajo colaborativo, obtener un resultado positivo que llenó de orgullo a sus realizadores, al punto que ellos mismos compartieron con la comunidad los avances en el proceso (Ver fig. 2)

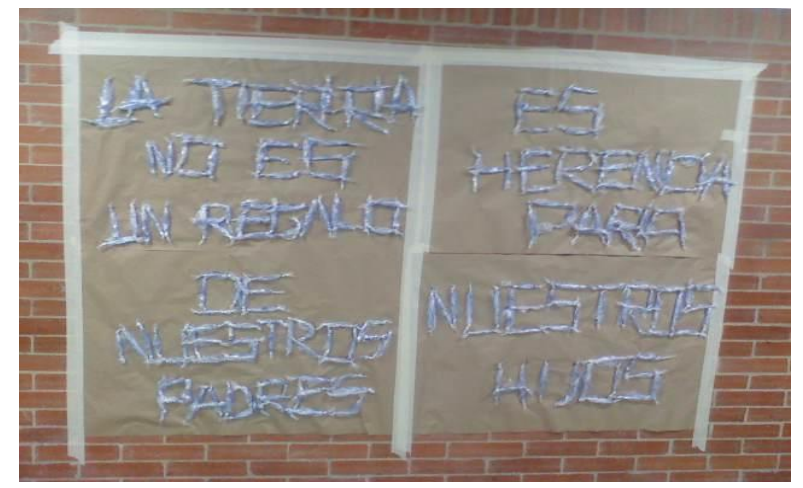

Fig 2. Cartelera elaborada por los estudiantes del grado 401

Esta primera actividad reforzó la intención de reutilizar el plástico. Posteriormente surgió la idea de elaborar un material que pudiera luego ser utilizado en el colegio. Nace la idea de construir ladrillos ecológicos, ya que éstos son elementos construidos con materiales que no degradan el medio ambiente y cuya fabricación también es respetuosa con este; frente a los ladrillos habituales cuya fabricación y materiales no es tan inocua, además de producir un ahorro energético posibilitan la reutilización de residuos durante su elaboración.

Los ladrillos elaborados por los estudiantes del IED utilizaron la botella de plástico PET resultante del consumo de gaseosas y jugos en la institución rellenas con el plástico en el que viene empacado el refrigerio. Estos ladrillos se caracterizan por ser duros, resistentes, aislantes, ligeros y económicos. Para implementar ésta idea, desde el aula, se originó un proceso de sensibilización en torno al significado del término basura; de las $3 R$ entendido como el proceso de disposición final de los recursos teniendo en cuenta Reducir, Reutilizar y/o Reciclar; así como el análisis del tiempo útil de los artefactos de uso cotidiano debido a la obsolescencia programada. 
Bio - grafia. Escritos sobre la Biología y su Enseñanza. ISSN 2027

Edición Extraordinaria. p.p. 744 - 770

Memorias del VIII Encuentro Nacional de Experiencias en Enseñanza de la Biología y la Educación Ambiental. III Congreso Nacional de Investigación en Enseñanza de la Biología.

Se compararon videos, alimentaron discusiones y como resultado de ésta actividad se concertó con los estudiantes la recolección de botellas de gaseosa tamaño familiar y del plástico resultante del refrigerio diario. Durante una sesión de clase a la semana con los estudiantes de grado cuarto y quinto se empezó el proceso de fabricación. La iniciativa fue acogida con entusiasmo y gran interés por parte de los estudiantes y pronto se convirtió en una de las sesiones favoritas de la semana.

Cabe aclarar que el desarrollo de éstas iniciativas surge de manera informal y como fruto de las inquietudes de las profesoras líderes del PRAE en el afán por responder a las inquietudes de los estudiantes y su necesidad manifiesta de involucrarse cada día más al proceso en forma personal. Como fruto de ésta idea inicial se finalizó el año escolar 2012 con un acopio de aproximadamente 500 ladrillos ecológicos. (Ver fig. 3)

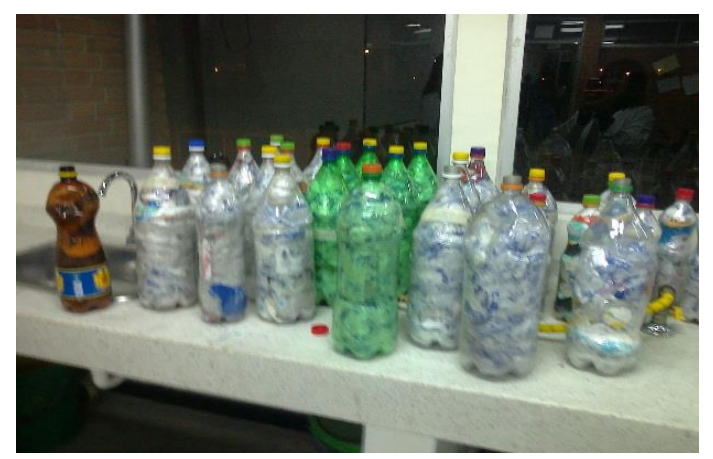

Fig. 3. Ladrillos ecológicos elaborados por los estudiantes

Finalizado el año 2012 y después de obtener un balance positivo en torno a la respuesta de los niños a las actividades planteadas en el tema ambiental, se consideró que era indispensable generar una serie de estrategias más puntuales que permitieran involucrar a toda la comunidad en el proceso de transformación de su realidad a partir de un trabajo concreto que mostrara resultados visibles. Por esto iniciando el año 2014 se generó un plan de acción para la Sede A, Jornada de la Tarde que permitiera desarrollar en los ciclos 3 y 4 las actividades del PRAE alrededor de tres ideas básicas:

- Que el proyecto diera respuesta a una necesidad de la Institución.

- Que la temática trabajada fuera incluida dentro del currículo del área de Ciencias.

- Que el producto contribuyera al embellecimiento del colegio. 
Bio - grafia. Escritos sobre la Biología y su Enseñanza. ISSN 2027

Edición Extraordinaria. p.p. 744 - 770

Memorias del VIII Encuentro Nacional de Experiencias en Enseñanza de la Biología y la Educación Ambiental. III Congreso Nacional de Investigación en Enseñanza de la Biología.

Con éste derrotero marcado. Se comenzó un proceso de gestión en forma personal, asumido por las profesoras encargadas del proyecto para buscar la ayuda pertinente que permitió darle luz verde a la iniciativa. De ésta forma fue posible hacer contacto con la Fundación Nápay quien en cabeza del señor Luis Andrés Sendoya, ofreció asesoría en la construcción de prototipos utilizando los ladrillos ecológicos montados el año anterior.

Simultáneamente y teniendo en cuenta la idea inicial de reutilización de residuos sólidos, se estableció también contacto con la Fundación del Pequeño Trabajador quien promueve desde hace 20 años el protagonismo y la organización de Niños, Niñas y Adolescentes Trabajadores (NAT's) de sectores populares en la ciudad de Bogotá; desde una perspectiva de reconocimiento como sujetos de derechos". Ellos han desarrollado un interesante trabajo con niños trabajadores del sector en el proceso de reciclaje de papel y elaboración de materiales sencillos para comercializar, además del acompañamiento en procesos de formación personal y de liderazgo. A partir de una visita a la Sede de la Fundación, se conocieron algunos de los niños participantes y con ellos se propuso un intercambio de saberes que beneficiaría a las dos instituciones.

En la misma vìa, se empezó el año escolar 2013 con el propósito de consolidar el Comité Ambiental Escolar CAE, que funciona como un estamento conformado por un grupo de estudiantes representantes de cada curso, es un espacio creado para la planeación y unificación de funciones y criterios en su labor de empoderamiento frente a su compromiso con el desarrollo del Proyecto Ambiental Institucional para la mejora de su ambiente (Concejo de Bogotá, 2005) . Para esto se planteó la idea en cada una de las aulas y se recibió una respuesta positiva. Los estudiantes se postularon para participar y una vez organizado el grupo de representantes de cada curso, se inició un proceso de "Formación en liderazgo basado en la Participación", ésta actividad es dirigida por la Fundación del Pequeño Trabajador quienes a través del Proyecto "El trabajo de Crecer" - que es desarrollado además en diferentes instituciones educativas de la localidad- y teniendo como base los Derechos Ambientales, impulsan a los niños a desplegar una labor basada en la autonomía y el compromiso personal y grupal para sacar adelante la iniciativa ambiental. (Ver figura 6)

Los niños del CAE, como fruto de ese proceso formativo, organizaron un Foro Ambiental Institucional (Ver figuras 5 y 6), una toma pacífica del colegio para hacer visible al Comité Ambiental en la Institución; también participaron en un Foro Interinstitucional en la Alcaldía Mayor de Bogotá con las instituciones copartícipes del Proyecto el "Trabajo de crecer" y en una sesión en el Congreso de la República. El común denominador de estas participaciones fue dar a 
Edición Extraordinaria. p.p. 744 - 770

Memorias del VIII Encuentro Nacional de Experiencias en Enseñanza de la Biología y la Educación Ambiental. III Congreso Nacional de Investigación en Enseñanza de la Biología.

conocer sus ideas y avances en la construcción de prototipos a la vez que resaltaron la importancia y el impacto que tiene su intervención en los procesos institucionales. Firman su "Manifiesto ecológico" y elaboran el símbolo por el cual fueran reconocidos como miembros del Comité. (Ver figura 7)

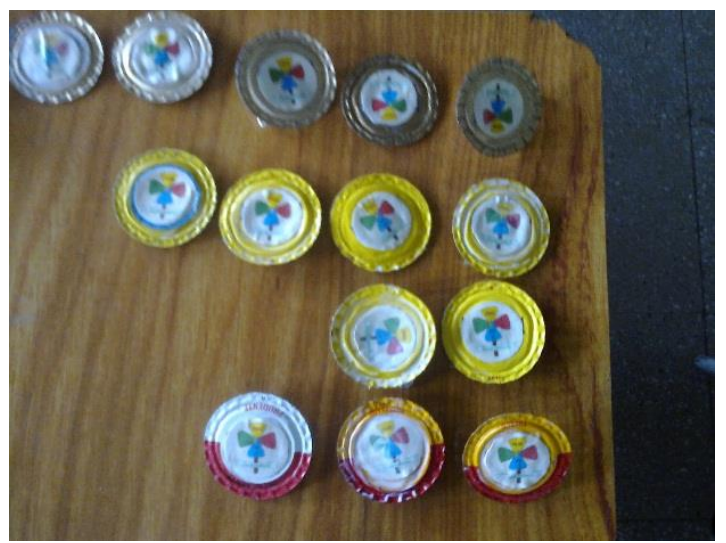

Fig. 5. Recordatorio del Foro Ambiental Institucional con el símbolo del comité, elaborado por los estudiantes.

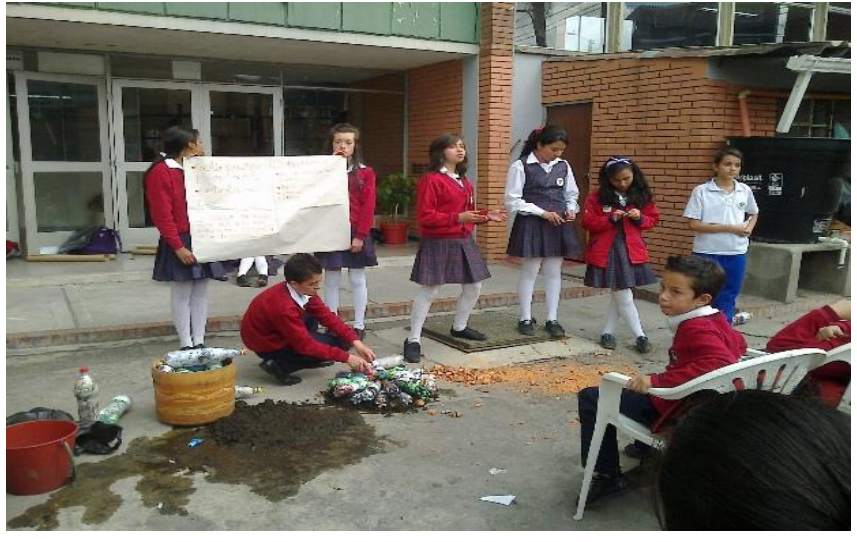

Fig. 6. Foro Ambiental

Con éstas actividades los niños empezaron a ser reconocidos a nivel institucional como gestores de cambio frente a la comunidad educativa. 
Bio - grafia. Escritos sobre la Biología y su Enseñanza. ISSN 2027

Edición Extraordinaria. p.p. 744 - 770

Memorias del VIII Encuentro Nacional de Experiencias en Enseñanza de la Biología y la Educación Ambiental. III Congreso Nacional de Investigación en Enseñanza de la Biología.

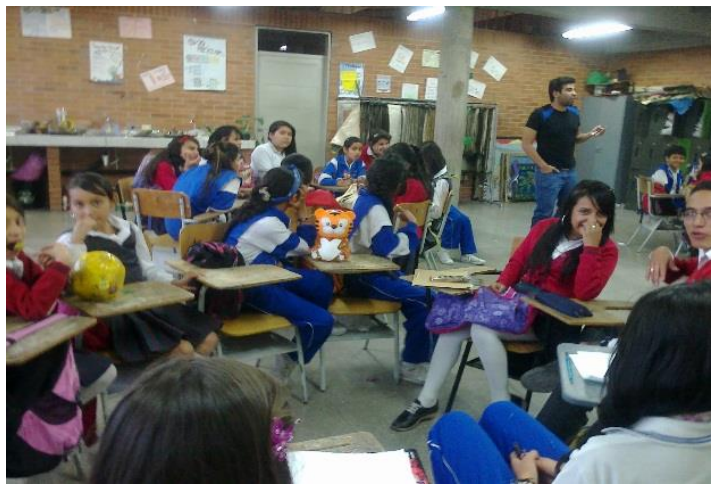

Fig. 6. Sesión del Comité Ambiental Escolar

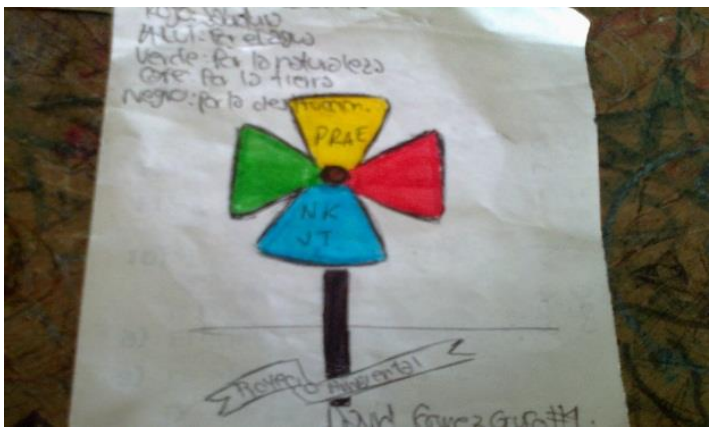

Fig. 7. Prototipo del símbolo del Comité Ambiental diseñado por los estudiantes en las mesas de trabajo

\section{Construcción de prototipos}

Denominamos prototipos al mobiliario ecológico construido por los estudiantes a partir de la utilización de materiales de desecho de la institución que serán la base para replicar la experiencia en el IED y en otras instituciones educativas que quieran conocer e implementar el proceso.

Siguiendo con la línea que fundamenta el PRAE, junto con los estudiantes de ciclo 4 la profesora Alicia González construyó una caneca (ver figura 8) para la recolección del plástico con los ladrillos ecológicos fabricados con anterioridad, cabe aclarar que el diseño y la mejora en el prototipo son el resultado de un trabajo creativo y colectivo fruto del ingenio de los estudiantes de este ciclo. En el proceso se involucró también al docente Braulio Moreno, licenciado en música y docente de ésta área en la jornada tarde, quien desde sus conocimientos artísticos participó en la asesoría de la decoración de la caneca con los niños de ciclo 3. 
Bio - grafia. Escritos sobre la Biología y su Enseñanza. ISSN 2027

Edición Extraordinaria. p.p. 744 - 770

Memorias del VIII Encuentro Nacional de Experiencias en Enseñanza de la Biología y la Educación Ambiental. III Congreso Nacional de Investigación en Enseñanza de la Biología.
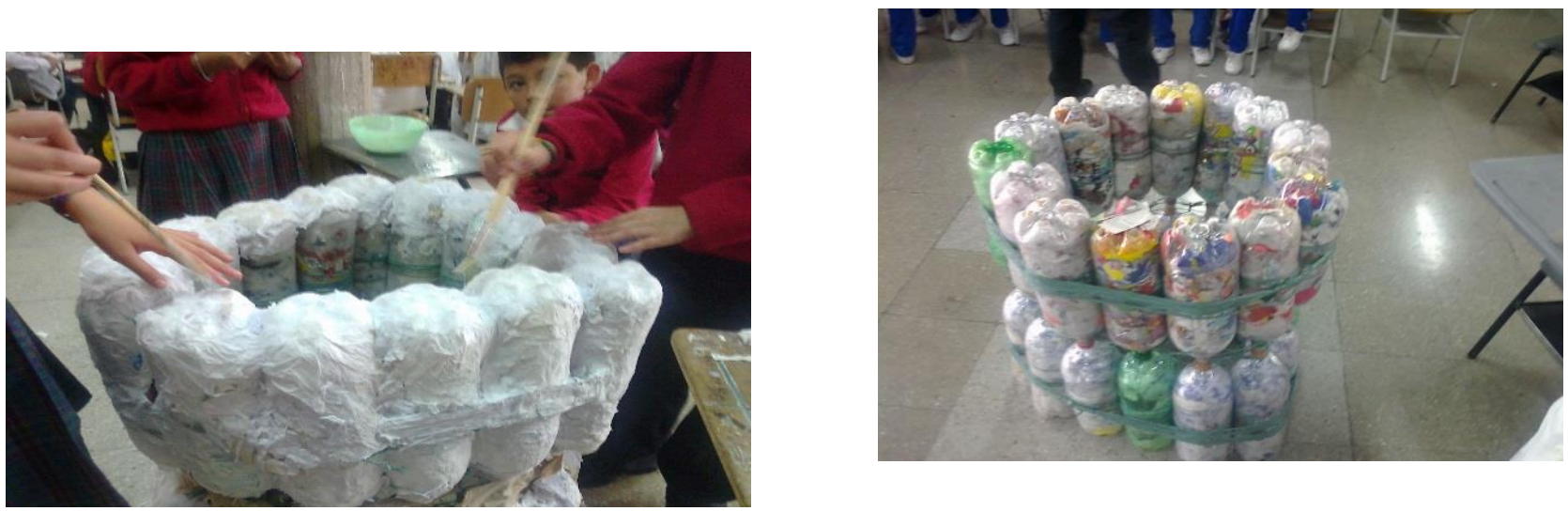

Fig. 8. Construcción de la caneca

\section{Resultados}

Teniendo como base este primer resultado y el compromiso adquirido por parte de los estudiantes del Comité Ambiental Escolar en la elaboración de prototipos que presten utilidad en el colegio, posteriormente, se levantó el diagnóstico de necesidades que permitiera escoger el mobiliario que será construido en la siguiente fase del proyecto.

A partir de la línea Gobernanza del agua planteada en el acuerdo 489 DE 2012 que propende por la búsqueda de una cultura ambiental en torno al agua para que la ciudadanía comprenda y actúe en procesos de adaptación frente al cambio climático (Concejo de Bogotá, D.C., 2012), surge la inquietud de fabricar un tanque de recolección y almacenamiento de agua lluvia que brinde disponibilidad de éste recurso. Su construcción se realizará con ladrillos ecológicos fabricados con botellas tamaño familiar y los responsables serán los gestores del ciclo 3 jornada mañana. El tanque estará ubicado en una zona estratégica del patio para tener acceso al agua durante el descanso y actividades deportivas.

Los estudiantes realizaron los cálculos pertinentes para el diseño del prototipo, llegando a la conclusión de que si el tanque tiene un diámetro y una altura de un metro, se necesitan cerca de 
Bio - grafia. Escritos sobre la Biología y su Enseñanza. ISSN 2027

Edición Extraordinaria. p.p. 744 - 770

Memorias del VIII Encuentro Nacional de Experiencias en Enseñanza de la Biología y la Educación Ambiental. III Congreso Nacional de Investigación en Enseñanza de la Biología.

2050 botellas, de las cuales hay fabricadas aproximadamente el 70\%. (Ver figura 9). Como esto no se ha realizado aún, además de la elaboración de los ladrillos y consecución de los demás materiales se adelanta un proceso de consulta por parte de los estudiantes en lo concerniente a las normas higiénicas y de bioseguridad que implica su implementación. Para la construcción del tanque se contará con la asesoría de un padre de familia que colaborará con su trabajo de albañilería.

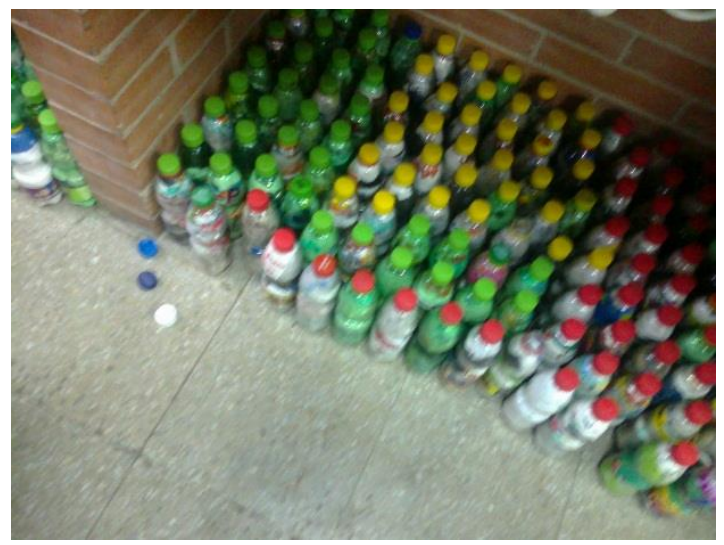

Fotografía 9. Botellas insumo para el Tanque de almacenamiento de agua lluvia

El segundo mueble prototipo a construir será una silla doble tipo tándem. Este prototipo se construirá con escombros utilizando la técnica de gavión, que es una caja rectangular o cuadrada elaborada en alambre y rellena de piedras o escombros. Para ello se utilizarán los escombros resultantes del cambio de la rejilla del borde del patio del colegio. Esta silla resulta de muy fácil construcción y bajo costo y resolvería la disponibilidad de muebles recreativos para usar en el patio de descanso.

El tercer ejercicio a realizar dadas las características de la planta física de la institución y la falta de plantas en las zonas comunes, es la construcción de muros verdes o jardines verticales utilizando diversas técnicas que tienen como base la reutilización de las botellas PET y en cuyo diseño aún se trabaja con los estudiantes de ciclo 4 de la Jornada de la Tarde. 
Bio - grafia. Escritos sobre la Biología y su Enseñanza. ISSN 2027

Edición Extraordinaria. p.p. 744 - 770

Memorias del VIII Encuentro Nacional de Experiencias en Enseñanza de la Biología y la Educación Ambiental. III Congreso Nacional de Investigación en Enseñanza de la Biología.

\section{Conclusiones}

La experiencia que se ha relatado anteriormente, es el fundamento de una línea de acción del PRAE del Colegio Fernando Soto Aparicio IED y ha sido de gran valor en los procesos de construcción de la cultura ambiental en los estudiantes de la institución. El empoderamiento de los estudiantes en las actividades del Comité Ambiental Escolar ha sido la base para liderar con autonomía los procesos con sus compañeros en el aula y presentar su experiencia en diferentes ámbitos académicos.

La constancia en la recolección de insumos y elaboración de los prototipos para el mobiliario, la responsabilidad con la que asumen sus encargos, la creatividad y trabajo colaborativo para superar las dificultades hace que sea una labor que involucra a los docentes más allá de su quehacer académico; es una labor que no tiene valoración sumativa, no se trabaja por un resultado en términos de notas para la valoración final en la asignatura, se trabaja con los estudiantes por los resultados, por el desarrollo de una meta más allá de la simple obligación de la implementación de un PRAE como requisito gubernamental e institucional. Se quiere formar en cultura ambiental, se quiere propender por comportamientos pro-ambientales que se trasciendan de la vida escolar a la vida familiar y ciudadana.

Esta experiencia es una apuesta para generar acciones que redunden en el fortalecimiento de la cultura ambiental a partir del desarrollo de estrategias que involucren a los estudiantes en los procesos de mejora de su entorno. La elaboración de prototipos en la escuela es un tema que abre posibilidades de construcción colectiva, de toma de conciencia en torno al cuidado del ambiente y a la reflexión sobre el manejo responsable de los residuos sólidos. 
Bio - grafia. Escritos sobre la Biología y su Enseñanza. ISSN 2027

Edición Extraordinaria. p.p. $743-757$

Memorias del VIII Encuentro Nacional de Experiencias en Enseñanza de la Biología y la Educación Ambiental. III Congreso Nacional de Investigación en Enseñanza de la Biología.

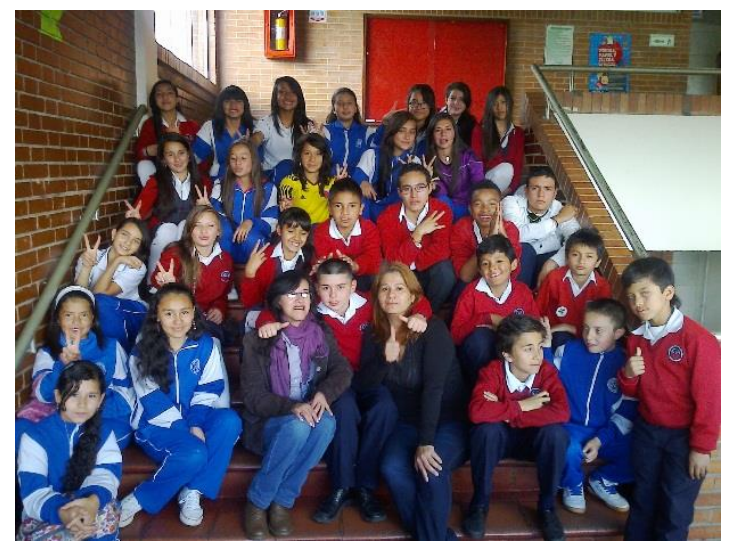

Comité Ambiental Escolar JT. Colegio Fernando Soto Aparicio IED.

\section{Referencias}

Alcaldia Mayor de Bogotá. (2011). Programa Basura Cero. Bogotá.

Colegio Fernando Soto Aparicio, IED. (2012). PROYECTO AMBIENTAL ESCOLAR, Por un ambiente que promueva el desarrollo de la naturaleza y la sociedad. Bogotá.

Concejo de Bogotá, D. (2005). Fortalecimiento de los Comités Ambientales Escolares. Bogotá.

Concejo de Bogotá, D.C. (12 de Junio de 2012). Acuerdo 489 DE 2012. Acuerdo 489 de 2012 Concejo de Bogotá D.C. Bogotá.

Miranda, L. (2013). Cultura ambiental: un estudio desde las dimensiones de valor, creencias, actitudes y comportamientos ambientales. . Producción más limpia. 
Bio - grafia. Escritos sobre la Biología y su Enseñanza. ISSN 2027

Edición Extraordinaria. p.p. 743 - 757

Memorias del VIII Encuentro Nacional de Experiencias en Enseñanza de la Biología y la Educación Ambiental. III Congreso Nacional de Investigación en Enseñanza de la Biología.

Red Internacional para la educación de la ciudadania y la transformación social. (Septiembre de 2015). Obtenido de http://www.redinternacionalepd.org/organizaciones-colombia.php 